\title{
Plant stanol ester consumption and arterial elasticity and endothelial function $\dagger$
}

\author{
Olli T. Raitakari ${ }^{1}$, Pia Salo $^{2}$, Helena Gylling ${ }^{3}$ and Tatu A. Miettinen ${ }^{4}$ \\ ${ }^{1}$ Department of Clinical Physiology, University of Turku, Turku, Finland \\ ${ }^{2}$ The Research Centre of Applied and Preventive Cardiovascular Medicine, University of Turku, Turku, Finland \\ ${ }^{3}$ Department of Clinical Nutrition, University of Kuopio, and Kuopio University Hospital, Kuopio, Finland \\ ${ }^{4}$ Department of Medicine, Division of Internal Medicine, University of Helsinki, Helsinki, Finland
}

(Received 1 August 2007 - Revised 12 December 2007 - Accepted 13 December 2007 - First published online 18 February 2008)

We evaluated whether plant stanol esters mixed with different vegetable oil spreads improved arterial health. A total of 200 adults with serum cholesterol $>5 \mathrm{mmol} / \mathrm{l}$ were randomised to consume camelina, rapeseed or sunflower oil spread with stanol ( $2 \mathrm{~g} / \mathrm{d})$ ester or sunflower oil spread without stanol ester (controls) for 3 months. Non-invasive ultrasound was used to measure carotid artery compliance (CAC) and brachial artery flow-mediated endothelial dependent vasodilatation (FMD) at baseline and after the intervention as markers of arterial health. Plant stanol esters reduced LDL-cholesterol by $9 \%$ compared with controls $(P<0.001)$ similarly in the different treatment groups. In the combined treatment groups ( $n$ 147), CAC or FMD were not changed from controls $(n$ 47). In a subgroup analysis, division of subjects at baseline into below and over sexspecific 50th percentiles of CAC and FMD revealed that low CAC was improved from 1.23 to $1.59 \%$ per $10 \mathrm{mmHg}$ in the treatment group ( $n$ 69), and from 1.42 to $1.47 \%$ per $10 \mathrm{mmHg}$ in controls ( $n 25),(P=0.0035$ between groups). Low FMD was improved from $6.9 \%$ to $8.6 \%$ in the treatment group ( $n$ 73) and from $6.6 \%$ to $6.8 \%$ in controls $(n 24)$ ( $P=0.05$ between groups). In the respective high-median groups, CAC and FMD were not changed in spite of significantly lowered LDL-cholesterol. In conclusion, consumption of plant stanol ester for 3 months had no overall significant effect on arterial elasticity and endothelial function. A controlled study is needed to test whether beneficial changes are obtained in subjects with initially reduced arterial elasticity and endothelial function.

Endothelial function: LDL-cholesterol: Plant stanol esters: Sitosterol

Ultrasonographically assessed changes in arterial function and structure are useful means to study early pathophysiological changes in arteries regarding the development of atherosclerosis. Carotid arterial compliance (CAC) or elasticity is a functional marker of arterial health. The compliance decreases as a consequence of many disease states such as atherosclerosis and diabetes ${ }^{(1)}$. In addition, risk factors, such as high LDLcholesterol level, elevated blood pressure, obesity and smoking, have been associated with decreased arterial elasticity in cross-sectional studies ${ }^{(1)}$. Impaired CAC has been implicated as an independent predictor for cardiovascular events in high-risk individuals ${ }^{(2)}$. Another commonly used marker of arterial health is endothelium-dependent brachial artery flowmediated dilatation (FMD) ${ }^{(3)}$. The dilatation response for increased blood flow is mainly mediated by nitric oxide released from arterial endothelial cells ${ }^{(4)}$. Brachial FMD response correlates with coronary endothelial function tested with invasive methods ${ }^{(5)}$ and it has been shown to predict cardiovascular events ${ }^{(6,7)}$.

Since increased LDL-cholesterol level is a major risk factor for the development of arterial atheromatosis, it is logical to consider that lowering of its level could improve abnormal ultrasonographic findings. In fact, lowering of
LDL-cholesterol with statins improves endothelial dysfunction $^{(8)}$ even with relatively low starting LDL-cholesterol levels ${ }^{(9)}$. However, lowering of LDL-cholesterol during inhibition of cholesterol absorption with ezetimibe alone or combined with statins is not consistently improving endothelial function ${ }^{(10)}$. Plant sterol and stanol esters lower LDL cholesterol by about $10 \%$ through inhibition of sterol absorption ${ }^{(11)}$, a change expected to reduce coronary events by $25 \%$ in longterm use ${ }^{(12)}$. Plant sterols alone or combined with dietary fibre did not, however, affect endothelial function despite a simultaneous marked LDL-cholesterol reduction ${ }^{(13)}$. Rapeseed oil plant stanol esters and soya oil plant sterol esters in a headto-head study lowered LDL-cholesterol similarly, but only the sterol esters decreased brachial artery diameter of mildly hypercholesterolaemic subjects ${ }^{(14)}$. The clinical relevance of this change, however, remains open. In children with familial hypercholesterolaemia, cholesterol lowering with plant sterol or stanol esters had no effect on endothelial function ${ }^{(15,16)}$. In view of the inconsistent effects of regular plant sterol or stanol ester consumption on arterial function, we studied the effects of plant stanol ester in spread with three different fatty acid compositions (camelina oil, rapeseed oil and sunflower oil) on serum lipids and endothelial function in

Abbreviations: CAC, carotid artery compliance; FMD, flow-mediated dilatation.

$\dagger$ Published partly as an abstract (Atherosclerosis (2007) 8(1), 167), 76th Congress of the European Atherosclerosis Society, 10-13 June 2007, Helsinki, Finland.

* Corresponding author: Dr Helena Gylling, fax +358 17162 792, email helena.gylling@uku.fi 
mildly hypercholesterolaemic subjects. For this purpose, a randomised, double-blind, placebo-controlled 3-month trial was performed to investigate whether plant stanol ester spread with different fatty acid compositions could improve arterial elasticity and endothelial function.

\section{Subjects and methods}

\section{Study population and design}

Study subjects were recruited from occupational health service clinic Mehiläinen in Turku, Finland. Information about the study was sent to several working places to be distributed amongst their employees. In addition, the subjects were recruited by advertising in a local newspaper. We invited otherwise healthy 20 to 50 year-old subjects with at least mild hypercholesterolaemia (total cholesterol $>5 \mathrm{mmol} / \mathrm{l}$ and serum TAG $<2.5 \mathrm{mmol} / \mathrm{l}$ ) to participate in the study. Total cholesterol concentration was verified before randomisation, unless a value of $>5 \mathrm{mmol} / \mathrm{l}$ had been documented within the previous 3 months or a value of $>5.4 \mathrm{mmol} / \mathrm{l}$ had been documented within the previous 6 months. The study subjects needed to be non-smokers (for at least 5 years), without cholesterol-lowering medication and without regular plant stanol/sterol use. Exclusion criteria also included the presence of severe obesity (BMI $>35 \mathrm{~kg} / \mathrm{m}^{2}$ ), diabetes, CVD, pregnancy or alcohol/drug abuse.

From among the visitors of the occupational health service, 200 subjects were recruited. They were randomised doubleblind to four groups (fifty in each), of which three consumed vegetable oil spread with plant stanol $(2 \mathrm{~g} / \mathrm{d})$ esters (active treatment groups 1-3), and the fourth without added plant stanol esters in the vegetable oil spread (control group). The 150 participants of the active treatment groups were matched with controls for age, serum and LDL-cholesterol level and CAC and FMD (all comparisons $P>0 \cdot 1$, data not shown). The intervention lasted for 3 months. The daily dose of spread was $25 \mathrm{~g}$. The active treatment group 1 consumed camelina oil spread with plant stanols transesterified with rapeseed oil fatty acids, group 2 consumed rapeseed oil spread with plant stanols transesterified with rapeseed oil fatty acids, group 3 had sunflower oil spread with plant stanols transesterified with rapeseed oil fatty acids and the control group 4 consumed sunflower oil spread without added plant stanol esters. The phytosterol composition of the stanol ester blend contained $88.1 \%$ sitostanol, $8.6 \%$ campestanol, $1.6 \%$ sitosterol and $0.7 \%$ other sterols. The spreads had a fat content of $50-51 \%$ (absorbable fat). The amount of saturated fatty acids was similar in the test spreads, $12-15 \mathrm{~g} / 100 \mathrm{~g}$ product: that of MUFA $18 \mathrm{~g} / 100 \mathrm{~g}$ in camelina oil spread, $27 \mathrm{~g} / 100 \mathrm{~g}$ in rapeseed oil spread and $15 \mathrm{~g} / 100 \mathrm{~g}$ in sunflower oil spreads: that of PUFA 19,12 and $22-24 \mathrm{~g} / 100 \mathrm{~g}$, respectively. The amount of trans fatty acids was $0 \cdot 1-0 \cdot 3 \mathrm{~g} / 100 \mathrm{~g}$ product.

Blood samples for lipid measurements were obtained after overnight fast at baseline and at the end simultaneously with body weight and ultrasound measurements. The participants were advised to continue their habitual home diet otherwise unchanged but to reduce their usual fat intake (about $23 \mathrm{~g}$ ). An estimated number of spread containers were divided to the participants at the beginning of each month and the intake of the spread was evaluated from the returned containers.

All subjects gave their written informed consent. The investigation was carried out in accordance with the principles of the Declaration of Helsinki. The study protocol was approved by the Joint Ethics Committee of the University of Turku and Turku University Central Hospital.

\section{Lipid measurements}

Fasting total and HDL-cholesterol and total TAG in serum were measured with routine methods with commercial kits and expressed in terms of mmol/l. LDL-cholesterol was calculated from the data ${ }^{(17)}$. Serum cholesterol, squalene and noncholesterol sterols, i.e. cholestenol, desmosterol, lathosterol (markers of cholesterol synthesis) ${ }^{(18)}$, and cholestanol, campesterol, sitosterol and avenasterol (markers of cholesterol absorption) ${ }^{(18,19)}$ were measured with a single gas-liquid chromatographic run from non-saponifiable serum lipids. GLC was performed with a $50 \mathrm{~m}$ long SE-30 capillary column (Ultra 2; Agilent Technologies, Wilmington, DE, USA) principally as shown earlier ${ }^{(20)}$. The values are given as ratios to cholesterol $\left(10^{2} \times \mathrm{mmol} / \mathrm{mol}\right.$ cholesterol $)$.

\section{Ultrasound studies}

We used Acuson Sequoia 512 mainframe (Mountain View, CA) with 8.0 MHz linear array transducer. Studies were performed after an overnight fast and after a 30 min rest. Subjects were instructed to discontinue any vasoactive medications $3 \mathrm{~d}$ before the study. The digitally stored scans were analysed by one reader blinded to subjects' details.

Carotid artery elasticity. To assess CAC, the best quality cardiac cycle was selected from the 5-s clip image. The common carotid diameter $10 \mathrm{~mm}$ from carotid bifurcation was measured from the B-mode images using ultrasonic callipers in end-diastole and end-systole, respectively. Brachial blood pressure was measured during the ultrasound study with an automated sphygmomanometer (Omron M4; Omron Matsusaka Co., Ltd., Japan). The ultrasound and concomitant brachial blood pressure measurements were used to calculate $\mathrm{CAC}$, as $([\mathrm{Ds}-\mathrm{Dd}] / \mathrm{Dd}) /(\mathrm{Ps}-\mathrm{Pd})$, where $\mathrm{Dd}$ is the diastolic diameter, Ds, the systolic diameter, Ps, systolic blood pressure and $\mathrm{Pd}$, diastolic blood pressure ${ }^{(21)}$. Thus, compliance is a marker of arterial elasticity, which measures the ability of the carotid arteries to expand as a response to pulse pressure caused by cardiac contraction and relaxation. We have previously reported $2.7 \%$ between-visit $\mathrm{CV}$ for carotid artery diastolic diameter measurements and $19.5 \%$ for $\mathrm{CAC}^{(21)}$.

Brachial artery test. Brachial artery diameter was measured as previously described ${ }^{(22)}$. In brief, a resting scan above the elbow was performed. Thereafter, hyperaemia was induced by inflation of a cuff placed around the forearm followed by release. Subsequent scans were taken at $40 \mathrm{~s}, 60 \mathrm{~s}$ and $80 \mathrm{~s}$ after the cuff release. FMD, as a marker of endothelial function, was calculated as the maximal percent increase in arterial diameter during hyperaemia compared with the resting value. This dilation response reflects endothelium-dependent vasorelaxation capacity because it can be blunted by simultaneous infusion of nitric oxide synthase inhibitor $^{(4)}$. We have previously reported $3.2 \%$ between-visit 
CV for brachial artery diameter measurements and $26.0 \%$ for $\mathrm{FMD}^{(22)}$

To evaluate whether the changes of CAC and FMD were related to their baseline values, the treatment and control groups were divided at baseline to below and above sexspecific 50th percentile of CAC and FMD.

\section{Statistical analysis}

The comparisons between the study groups were performed using ANOVA with Bonferroni correction, two-sided $t$ test and paired $t$ test. Correlation coefficients were calculated with Spearman rank correlations. A $P$ value $<0.05$ was considered statistically significant.

\section{Results}

Table 1 presents the baseline characteristics of the combined actively treated groups and controls. Seven subjects from the 150 active treatment group participants and three of the fifty controls interrupted the study during the 3-month period for reasons not related to the investigation, so that 143 and forty-seven subjects completed the present study. The compliance of consuming the spreads was 96 (SE 0.01) \% in the active treatment and 97 (SE 0.01) \% in the control group. Body weight was unchanged in all groups during the intervention.

\section{Lipids and sterols}

Plant stanol ester intake reduced LDL-cholesterol concentration by $6 \%$ from baseline $(P<0.001)$. This treatment effect did not differ across the three active treatment groups $(P=0 \cdot 14)$ indicating that the 143 stanol-treated subjects could be combined to a single group (Table 2). In controls,

Table 1. Baseline characteristics of the study subjects $\dagger$

(Mean values with their standard errors)

\begin{tabular}{|c|c|c|c|c|}
\hline \multirow[b]{2}{*}{ Variables } & \multicolumn{2}{|c|}{$\begin{array}{l}\text { Treatment } \\
\text { group }\end{array}$} & \multicolumn{2}{|c|}{ Control group } \\
\hline & Mean & SE & Mean & SE \\
\hline $\begin{array}{l}\text { No. } \\
\text { Males }\end{array}$ & 143 & & 47 & \\
\hline$n$ & 68 & & 32 & \\
\hline$\%$ & 45 & & 64 & \\
\hline Age (years) & $42 \cdot 5$ & 0.5 & $42 \cdot 2$ & 0.8 \\
\hline BMI $\left(\mathrm{kg} / \mathrm{m}^{2}\right)$ & $25 \cdot 4^{*}$ & 0.3 & $27 \cdot 0$ & 0.3 \\
\hline Waist circumference $(\mathrm{cm})$ & $88^{*}$ & 1 & 92 & 1 \\
\hline Systolic blood pressure (mmHg) & 130 & 1 & 133 & 2 \\
\hline Diastolic blood pressure $(\mathrm{mmHg})$ & 80 & 1 & 81 & 1 \\
\hline Glucose $(\mathrm{mmol} / \mathrm{l})$ & 5.46 & 0.03 & $5 \cdot 51$ & 0.06 \\
\hline Serum cholesterol (mmol/l) & $5 \cdot 83$ & 0.07 & $5 \cdot 95$ & $0 \cdot 14$ \\
\hline LDL-cholesterol (mmol/l) & 3.47 & 0.06 & $3 \cdot 80$ & 0.14 \\
\hline HDL-cholesterol (mmol/l) & $1 \cdot 71$ & 0.04 & $1 \cdot 62$ & 0.05 \\
\hline Serum TAG (mmol/l) & $1 \cdot 39$ & 0.07 & 1.28 & 0.11 \\
\hline CAC (\%/10 mmHg) & 1.78 & 0.06 & $1 \cdot 72$ & 0.06 \\
\hline FMD (\%) & $10 \cdot 8$ & 0.5 & $9 \cdot 8$ & 0.7 \\
\hline
\end{tabular}

CAC, carotid artery compliance; FMD, brachial artery flow-mediated dilatation.

Mean values were significantly different from control: ${ }^{*} P<0.05$

†For details of subjects and procedures, see Subjects and methods.
LDL-cholesterol increased by $3.6 \%$. Thus, there was a significant $9.3 \%$ difference between the intervention and control groups $(P=0 \cdot 003)$. There was no change in HDL-cholesterol and serum TAG levels (data not shown).

Cholesterol synthesis was increased with plant stanol ester consumption, as shown for cholestenol and lathosterol ratios to cholesterol by about $20 \% v$. baseline and by about $25 \%$ $v$. controls $(P<0.01$ for both, Table 2). The absorption sterol ratios were decreased, as shown for campesterol and sitosterol by up to $27 \% v$. baseline and by $35 \% v$. controls $(P<0.001$ for both $)$.

\section{Arterial elasticity and endothelial function}

The intervention was not associated with significant absolute changes in CAC or FMD (Table 2). A further subgroup analysis was performed to test the (post hoc) hypothesis that subjects with below median arterial elasticity or endothelial function values might respond differently to treatment compared with subjects with values above median.

Below-median carotid artery compliance. In subjects with low baseline CAC ( $n$ 95), the intervention in the active treatment group ( $n$ 69) significantly reduced total cholesterol from baseline, but not from controls (Table 3). Total cholesterol was significantly reduced from baseline $(P<0.001)$, but LDL-cholesterol reduction was not significant $(P=0.056)$. The respective ratios of the synthesis markers cholestenol and lathosterol were increased and those of absorption markers cholestanol, campesterol, sitosterol and avenasterol were decreased both from baseline and from controls $(P<0.01$ for all, except cholestanol $v$. controls).

The average CAC changed from 1.23 (SE 0.04) to 1.59 (SE 0.08$) \% / 10 \mathrm{mmHg}$ in the active treatment group ( $n$ 69), and from 1.42 (SE 0.05) to 1.47 (SE 0.07$) \% / 10 \mathrm{mmHg}$ in controls ( $n$ 25), $(P=0.004$ between groups).

Above-median carotid artery compliance. In subjects with high baseline CAC ( $n$ 95), both total and LDL-cholesterol levels were decreased in the active treatment group ( $n$ 74) as compared with baseline or control levels $(P<0.05$, Table 3). Percentage changes of squalene, cholestenol and lathosterol ratios were increased with plant stanol esters from baseline and from controls $(P<0.01$ for all). All absorption marker ratios including cholestanol were significantly decreased with the active treatment from baseline and from controls $(P<0.05$ for all $)$.

The average CAC changed from 2.32 (SE 0.05) to 1.97 (SE 0.07) $\% / 10 \mathrm{mmHg}$ in the active treatment group ( $n$ 72) and from 2.09 (SE 0.07) to 1.94 (SE 0.15) $\% / 10 \mathrm{mmHg}$ in controls ( $n$ 21) (NS between groups).

Below- and above-median flow-mediated dilatation. In subjects with low FMD, the average FMD changed from 6.87 (SE 0.37 ) to 8.85 (SE 0.63 ) $\%$ in the active treatment group ( $n$ 73), and from 6.63 (SE 0.60) to 6.77 (SE 0.73) \% in controls ( $n$ 24), $(P=0.05$ between the groups). In subjects with high FMD, the respective values were from 14.96 (SE 0.63) to 12.93 (SE 0.86) \% in the active treatment group ( $n$ 69) and from 13.02 (SE 0.92) to 11.07 (SE 1.04) \% in controls ( $n$ 23) (NS between groups). The lipid and sterol changes were essentially similar to those observed with low and high CAC (data not shown). 
Table 2. Effects of plant stanol ester on LDL-cholesterol level, non-cholesterol sterol ratios to cholesterol, carotid artery compliance (CAC) and brachial artery flow-mediated endothelial- dependent vasodilatation (FMD)*

(Mean values with their standard errors)

\begin{tabular}{|c|c|c|c|c|c|}
\hline \multirow[b]{2}{*}{ Variables } & & \multicolumn{2}{|c|}{$\begin{array}{l}\text { Treatment group } \\
\qquad(n 143)\end{array}$} & \multicolumn{2}{|c|}{$\begin{array}{l}\text { Control group } \\
\quad(n 47)\end{array}$} \\
\hline & & Mean & SE & Mean & SE \\
\hline \multirow[t]{2}{*}{ LDL-cholesterol (mmol/l) } & Baseline & 3.47 & 0.06 & $3 \cdot 80$ & 0.14 \\
\hline & Change, \% & $-5 \cdot 66 \dagger \ddagger$ & 1.42 & $3 \cdot 62$ & $2 \cdot 32$ \\
\hline \multirow[t]{2}{*}{ Cholestenol§ } & Baseline & $19 \cdot 0$ & 0.6 & $20 \cdot 3$ & 1.0 \\
\hline & Change, \% & 18.8†‡ & $2 \cdot 1$ & -0.5 & $3 \cdot 7$ \\
\hline \multirow[t]{2}{*}{ Lathosterol§ } & Baseline & $131 \cdot 7$ & $4 \cdot 2$ & $136 \cdot 6$ & $7 \cdot 0$ \\
\hline & Change, \% & 21.3†‡ & $2 \cdot 5$ & $2 \cdot 7$ & $3 \cdot 4$ \\
\hline \multirow[t]{2}{*}{ Campesterol§ } & Baseline & 274.3 & $9 \cdot 1$ & $286 \cdot 9$ & $15 \cdot 7$ \\
\hline & Change, \% & $-26 \cdot 3 † \ddagger$ & $1 \cdot 3$ & $2 \cdot 3$ & $3 \cdot 3$ \\
\hline \multirow[t]{2}{*}{ Sitosterol§ } & Baseline & $129 \cdot 2$ & 4.9 & $137 \cdot 5$ & $6 \cdot 8$ \\
\hline & Change, \% & $-26 \cdot 7 \dagger \ddagger$ & $1 \cdot 3$ & 5.4 & $2 \cdot 7$ \\
\hline \multirow[t]{2}{*}{$\mathrm{CAC}(\% / 10 \mathrm{mmHg})$} & Baseline & 1.78 & 0.06 & $1 \cdot 72$ & 0.06 \\
\hline & Change & 0.002 & 0.706 & -0.041 & 0.587 \\
\hline \multirow[t]{2}{*}{ FMD (\%) } & Baseline & $10 \cdot 80$ & 0.49 & $9 \cdot 76$ & 0.72 \\
\hline & Change & 0.03 & $6 \cdot 13$ & -0.88 & 3.78 \\
\hline
\end{tabular}

* For details of subjects and procedures, see Subjects and methods.

† Mean values were significantly different from baseline $(P<0.05)$

$\ddagger$ Mean values were significantly different from controls $(P<0.05)$

$\S 10^{2} \mathrm{mmol} / \mathrm{mol}$ cholesterol.

\section{Discussion}

These data show that a daily intake of $2 \mathrm{~g}$ plant stanols from stanol ester spread with different fatty acid composition lowers LDL-cholesterol over $9 \%$ compared with controls in a 3-month intervention. This effect was similar across the different spread formulae, indicating that the different fatty acid composition of the camelina oil, rapeseed oil and sunflower oil spreads did not differently affect the cholesterollowering effect of plant stanols, even when compared with

Table 3. Serum total and LDL-cholesterol, squalene and non-cholesterol sterol ratios to cholesterol at baseline and \% change after the intervention in subjects divided at baseline into below (low) and above (high) mean of baseline carotid artery compliance (CAC) ${ }^{\star}$

(Mean values with their standard errors)

\begin{tabular}{|c|c|c|c|c|c|c|c|c|c|}
\hline \multirow[b]{3}{*}{ Variables } & & \multicolumn{4}{|c|}{ Low CAC (n95) } & \multicolumn{4}{|c|}{ High CAC ( $n$ 95) } \\
\hline & & \multicolumn{2}{|c|}{ Treatment (n 69) } & \multicolumn{2}{|c|}{ Control (n 26) } & \multicolumn{2}{|c|}{ Treatment (n 74) } & \multicolumn{2}{|c|}{ Control ( $n$ 21) } \\
\hline & & Mean & SE & Mean & SE & Mean & SE & Mean & SE \\
\hline \multirow[t]{2}{*}{ Cholesterol (mmol/l) } & Baseline & $5 \cdot 9$ & $0 \cdot 1$ & $6 \cdot 1$ & 0.2 & $5 \cdot 7$ & $0 \cdot 1$ & $5 \cdot 7$ & $0 \cdot 2$ \\
\hline & Change, \% & -4.9 & $1 \cdot 2$ & -1.4 & 1.9 & $-3.9+$ & $1 \cdot 3$ & $6 \cdot 4$ & $2 \cdot 8$ \\
\hline \multirow[t]{2}{*}{ LDL- cholesterol (mmol/l) } & Baseline & 3.4 & 0.1 & $4 \cdot 0$ & 0.2 & 3.5 & $0 \cdot 1$ & $3 \cdot 6$ & 0.2 \\
\hline & Change, \% & $-4 \cdot 4$ & $2 \cdot 2$ & -0.5 & $2 \cdot 3$ & $-6.9 \dagger$ & $1 \cdot 8$ & $8 \cdot 7$ & $4 \cdot 2$ \\
\hline \multirow[t]{2}{*}{ Squalene $\ddagger$} & Baseline & 21 & 2 & 17 & 2 & 16 & 1 & 18 & 2 \\
\hline & Change, \% & 12 & 8 & 21 & 11 & $19 \dagger$ & 6 & -3 & 7 \\
\hline \multirow[t]{2}{*}{ Cholestenolf } & Baseline & 19 & 1 & 21 & 1 & 19 & 1 & 20 & 1 \\
\hline & Change, \% & $22 \dagger$ & 3 & -4 & 6 & $16 \dagger$ & 3 & 4 & 4 \\
\hline \multirow[t]{2}{*}{ Desmosterolł } & Baseline & 96 & 3 & 99 & 4 & 94 & 2 & 93 & 4 \\
\hline & Change, \% & 8 & 1 & 3 & 3 & 5 & 1 & 5 & 3 \\
\hline \multirow[t]{2}{*}{ Lathosterolf } & Baseline & 133 & 6 & 136 & 8 & 130 & 6 & 138 & 13 \\
\hline & Change, \% & $24 \dagger$ & 4 & -0.4 & 5 & 19† & 3 & 6 & 4 \\
\hline \multirow[t]{2}{*}{ Campesterolł } & Baseline & 281 & 14 & 277 & 21 & 268 & 12 & 299 & 25 \\
\hline & Change, \% & $-28 \dagger$ & 2 & 4 & 5 & $-25 \dagger$ & 2 & -0.2 & 3 \\
\hline \multirow[t]{2}{*}{ Sitosterolł } & Baseline & 135 & 8 & 138 & 9 & 124 & 6 & 137 & 11 \\
\hline & Change, \% & $-28 \dagger$ & 2 & 5 & 4 & $-26 \dagger$ & 2 & 6 & 3 \\
\hline \multirow[t]{2}{*}{ Avenasterol $\ddagger$} & Baseline & 46 & 2 & 46 & 2 & 44 & 1 & 47 & 3 \\
\hline & Change, \% & $-18 \dagger$ & 2 & 4 & 4 & $-16 \dagger$ & 2 & 5 & 4 \\
\hline \multirow[t]{2}{*}{ Cholestanol } & Baseline & 149 & 4 & 160 & 7 & 145 & 3 & 150 & 6 \\
\hline & Change, \% & -6 & 1 & -2 & 2 & $-6 \dagger$ & 1 & -1 & 1 \\
\hline
\end{tabular}

${ }^{*}$ For details of subjects and procedures, see Subjects and methods.

$\dagger$ Mean values were significantly different from controls $(P<0.05)$

$\ddagger 10^{2} \mathrm{mmol} / \mathrm{mol}$ cholesterol. 
baseline or with sunflower oil control spread. Second, the data suggested that the intake of plant stanol esters did not affect elasticity of carotid artery and endothelial function in the whole treatment group in spite of serum total and LDL-cholesterol lowering.

Serum cholesterol reduction by statins has been reported to improve endothelial function in children with familial hypercholesterolaemia ${ }^{(8)}$ or in normocholesterolaemic subjects ${ }^{(9)}$. Statins effectively reduce cholesterol synthesis, but increase serum plant sterol levels ${ }^{(23)}$, while ezetimibe induces cholesterol malabsorption like plant stanols and reduces cholesterol and plant sterol absorption and increases cholesterol synthesis ${ }^{(24)}$. However, ezetimibe alone or even in combination with statin does not consistently improve endothelial function $^{(10)}$. In previous studies, consumption of plant sterols and stanols for a relatively short time significantly reduced serum cholesterol, but had no consistent effect on FMD in children with familial hypercholesterolaemia ${ }^{(15,16)}$. Respective statin treatment, however, improved FMD probably mostly due to a more effective reduction of LDL-cholesterol ${ }^{(8)}$. Head-to-head comparison of plant stanol and sterol esters with a crossover design and each consumed for 10 weeks was carried out in moderately hypercholesterolaemic subjects comparing the values with each other and a matched control group $^{(14)}$. It was concluded that even though LDL-cholesterol was similarly decreased by the two spreads, sterol ester reduced the brachial artery diameter as compared with stanol esters with no virtual difference in $\mathrm{FMD}^{(14)}$. De Jong et al. ${ }^{(25)}$ recently investigated the effects of long-term plant sterol or stanol ester consumption on endothelial function and arterial stiffness in patients on statin treatment. Overall, they found no effect on vascular function, but observed in a subgroup analysis of their data that plant sterols and stanols improved endothelial dysfunction and arterial stiffness in patients at risk for CVD, indicated by high baseline levels of matrix metalloproteinase-9. A subgroup analysis of the present study similarly showed that arterial elasticity and endothelial function were improved only in subjects who had below average baseline values for these vascular parameters. Obviously, the results of these post hoc analyses must be interpreted cautiously. Nevertheless, they raise the possibility that plant stanols/sterols may improve vascular function in subjects with suboptimal values. However, a controlled study is needed to test this hypothesis.

Even though the present results can only be considered as hypothesis generating, it can be assumed that in subjects with low baseline endothelial function, as compared with those with the high respective one, the endothelial layer of arteries is thicker owing to excessively precipitated circulating lipid material and that lowering of blood lipids could then improve the endothelial function. The relatively small LDL reduction in the low baseline endothelial function group, however, was significantly associated with the improved endothelial function. It should be borne in mind that statin-induced improvement of endothelial function is not consistently associated with lipid-lowering, but it can be related to pleiotropic function of statin therapy ${ }^{(10)}$. Since plant stanols are virtually unabsorbable, their pleiotropic function can hardly be considered. Additional studies of the present population indicated that baseline serum cholesterol or baseline and follow-up HDL-cholesterol and serum TAG levels, blood pressure and
BMI were not related to endothelial function or the changes observed within it.

Our observations on measurement of non-cholesterol sterols also showed a marked reduction in serum plant sterols and an increase in cholesterol synthesis with plant stanols. This would theoretically also reduce endothelial plant sterol contents, the role of which is not known in the regulation of endothelial function. The changes of serum plant sterols were, however, similar in the subgroups with high and low endothelial function.

In conclusion, 3-month consumption of plant stanol ester spread did not affect endothelial function in the whole population. An increase in arterial elasticity and endothelial reactivity in subjects with below average baseline values raises the possibility that plant stanols may improve vascular function in subjects with suboptimal values.

\section{Acknowledgements}

We thank Eija Piispa for preparing the test spreads. Raisio Benecol Ltd, Raisio, Finland supported the study with a grant. Pia Salo is a medical adviser at Raisio Benecol Ltd.

\section{References}

1. Zieman SJ, Melenovsky V \& Kass DA (2005) Mechanisms, pathophysiology, and therapy of arterial stiffness. Arterioscler Thromb Vasc Biol 25, 932-943.

2. Blacher J, Pannier B, Guerin AP, Marchais SJ, Safar ME \& London GM (1998) Carotid arterial stiffness as a predictor of cardiovascular and all-cause mortality in end-stage renal disease. Hypertension 32, 570-574.

3. Celermajer DS, Sorensen KE, Gooch VM, Spiegelhalter DJ, Miller OI, Sullivan ID, Lloyd JK \& Deanfield JE (1992) Noninvasive detection of endothelial dysfunction in children and adults at risk of atherosclerosis. Lancet 340, 1111-1115.

4. Mullen MJ, Kharbanda RK, Cross J, Donald AE, Taylor M, Vallance P, Deanfield JE \& MacAllister RJ (2001) Heterogenous nature of flow-mediated dilatation in human conduit arteries in vivo: relevance to endothelial dysfunction in hypercholesterolemia. Circ Res 88, 145-151.

5. Anderson TJ, Uehata A, Gerhard MD, et al. (1995) Close relation of endothelial function in the human coronary and peripheral circulations. J Am Coll Cardiol 26, 1235-1241.

6. Chan SY, Mancini GB, Kuramoto L, Schulzer M, Frohlich J \& Ignaszewski A (2003) The prognostic importance of endothelial dysfunction and carotid atheroma burden in patients with coronary artery disease. J Am Coll Cardiol 42, 1037-1043.

7. Gokce N, Keaney JF Jr, Hunter LM, Watkins MT, Nedeljkovic ZS, Menzoian JO \& Vita JA (2003) Predictive value of noninvasively determined endothelial dysfunction for long-term cardiovascular events in patients with peripheral vascular disease. J Am Coll Cardiol 41, 1769-1775.

8. de Jongh S, Lilien MR, op't Roodt J, Stroes ES, Backer HD \& Kastelein JJ (2002) Early statin therapy restores endothelial function in children with familial hypercholesterolemia. J Am Coll Cardiol 40, 2117-2121.

9. Hsu HY, Wang PY, Chen YT, Sheu WH, Hu HH \& Sheng WY (2005) Changes in flow-mediate dilatation, cytokines and carotid arterial stenosis during aggressive atorvastatin treatment in normocholesterolemic patients. J Chin Med Assoc 68, 53-68.

10. Fichtlscherer S, Schmidt-Lucke C, Bojunga S, Rossig L, Heeschen C, Dimmeler S \& Zeiher AM (2006) Differential 
effects of short-term lipid lowering with ezetimibe and statins on endothelial function in patients with CAD: clinical evidence for 'pleiotropic' functions of statin therapy. Eur Heart $J$ 27, $1182-1190$.

11. Katan MB, Grundy SM, Jones P, Law M, Miettinen $\mathrm{T}$ \& Paoletti R (2003) Efficacy and safety of plant stanols and sterols in the management of blood cholesterol levels. Mayo Clin Proc 78, 965-978

12. Law M (2000) Plant sterol and stanol margarines and health. BMJ 320, 861-864.

13. Ambring A, Friberg P, Axelsen M, Laffrenzen M, Taskinen MR, Basu S \& Johansson M (2004) Effects of a Mediterranean-inspired diet on blood lipids, vascular function and oxidative stress in healthy subjects. Clin Sci 106, 519-525.

14. Hallikainen M, Lyyra-Laitinen T, Laitinen T, Pihlajamäki J, Rauramaa R, Miettinen TA \& Gylling H (2006) Endothelial function in hypercholesterolemic subjects: effects of plant stanol and sterol esters. Atherosclerosis 188, 425-432.

15. de Jongh S, Vissers MN, Rol P, Backer HD, Kastelein JJ \& Stroes ES (2003) Plant sterols lower LDL cholesterol without improving endothelial function in prepubertal children with familial hypercholesterolaemia. J Inherit Metab Dis 26, 343-351.

16. Jakulj L, Vissers MN, Rodenburg J, Wiegman A, Trip MD \& Kastelein JJ (2006) Plant stanols do not restore endothelial function in pre-pubertal children with familial hypercholesterolemia despite reduction of low-density lipoprotein cholesterol levels. J Pediatr 148, 495-500.

17. Friedewald WT, Levy RI \& Fredrickson DS (1972) Estimation of the concentration of low-density lipoprotein cholesterol in plasma, without use of the preparative ultracentrifuge. Clin Chem 18, 499-502.
18. Miettinen TA, Tilvis RS \& Kesäniemi YA (1990) Serum plant sterols and cholesterol precursors reflect cholesterol absorption and synthesis in volunteers of a randomly selected male population. Am J Epidemiol 131, 20-31.

19. Miettinen TA, Tilvis RS \& Kesäniemi YA (1989) Serum cholestanol and plant sterol levels in relation to cholesterol metabolism in middle-aged men. Metabolism 38, 136-140.

20. Miettinen TA (1988) Cholesterol metabolism during ketoconazole treatment in man. $J$ Lipid Res 29, 43-51.

21. Juonala M, Järvisalo MJ, Mäki-Torkko N, Kähönen M, Viikari JS \& Raitakari OT (2005) Risk factors identified in childhood and decreased carotid artery elasticity in adulthood. The cardiovascular risk in young Finns study. Circulation 112, 1486-1493.

22. Juonala M, Viikari JS, Laitinen T, Marniemi J, Helenius H, Rönnemaa T \& Raitakari OT (2004) Interrelations between brachial endothelial function and carotid intima-media thickness in young adults: the cardiovascular risk in young Finns study. Circulation 110, 2918-2923.

23. Miettinen TA, Strandberg T \& Gylling H, for the Finnish investigators of Scandinavian Simvastatin Survival Study Group (2000) Noncholesterol sterols and cholesterol lowering by long-term simvastatin treatment in coronary patients, relation to basal serum cholestanol. Arterioscler Thromb Vasc Biol 20, $1340-1346$.

24. Sudhop T, Lütjohann D, Kodal A, Igel M, Tribble DL, Shah S, Perevozskaya I \& von Bergmann K (2002) Inhibition of cholesterol absorption by ezetimibe in humans. Circulation 106, 1943-1948.

25. de Jong A, Plat J, Hoeks AP \& Mensink RP (2007) Effects of long-term plant sterol or stanol ester consumption on endothelial function and arterial stiffness in patients on statin treatment. Atherosclerosis 8/1, 1 (Abstract). 